\title{
Differentiation among Young and Market-ready Cabernet Sauvignon, Pinotage and Shiraz Wines: Application of Canonical Discriminant Analysis using Flavonoid and Non-Flavonoid Compositional Data
}

\author{
P.P. Minnaar ${ }^{*}$ and M. Booyse ${ }^{2}$ \\ (1) ARC Infruitec-Nietvoorbija , Private Bag X5026, Stellenbosch 7600, South Africa \\ (2) ARC Biometry Unit, Private Bag X5026, Stellenbosch, Stellenbosch 7600, South Africa
}

Submitted for publication: September 2010

Accepted for publication: May 2011

Key words: Flavanols, flavonols, phenolic acids, anthocyanins, grape cultivar

\begin{abstract}
Various factors affect the polyphenol compound concentrations of red grapes. These include cultivar, vineyard location, viticultural practices, microclimate, soil type and winemaking processes. Polyphenol compound concentrations of young and market-ready Cabernet Sauvignon, Pinotage and Shiraz wines were examined for the purpose of discrimination between grape cultivars. Thirty-five individual polyphenol compounds were quantified using a HPLC-DAD method. Stepwise discriminant analysis (SDA) was used to select a subset of discriminatory variables. In addition, data was grouped and investigated by canonical discriminant analysis, which showed that polyphenol concentrations can be used to discriminate among grape cultivars, young wines and market-ready wines. Flavonol, flavanol and anthocyanin patterns were used as a basis for differentiation of young wines, while flavanols and phenolic acids were used for differentiation of market-ready wines. Discriminant analysis performed at $95 \%$ significance level revealed a $\mathbf{1 0 0 \%}$ categorisation of market-ready and young wines in terms of cultivar and $77.85 \%$ categorisation of a combination of market-ready and young wines in terms of grape cultivar. This illustrates the validity of polyphenols for studies pertaining to grape cultivar discrimination.
\end{abstract}

\section{INTRODUCTION}

Qualitative and quantitative phenolic composition of grapes depend on factors such as vineyard location, climate (including micro-climate), grape cultivar, soil type and degree of ripeness (Ferrer-Gallego et al., 2010). The two principal classes of polyphenol compounds present in grapes are the flavonoids and non-flavonoids. Non-flavonoids, which arise primarily from grape juice, mainly consist of hydroxycinnamic and benzoic acids. Flavonoids, which are localised in the skins and seeds of grapes, consist mainly of flavonols [kaempferol, quercetin and myricetin], flavanols $[(+)$-catechins and (-)-epicatechins], flavones [2 phenyl chromone nucleus] and anthocyanins (Zoecklein, et al., 1999). The most important polyphenol compounds in red grapes and wine are anthocyanins, flavanols and flavonols (Ribéreau-Gayon et al., 2006).

The same polyphenol compounds occur in different grape cultivars, although the relative concentrations of the individual compounds differ considerably among cultivars (Burns et al., 2002; Rossouw \& Marais, 2004; Makris et al., 2006; Ribéreau-Gayon et al., 2006). Polyphenol compound concentrations can be used as an analytical tool to characterise and differentiate among grape cultivars and for the classification of wines (Revilla et al., 2001; GarciaBeneytez et al., 2003; De Villiers et al., 2005; Rastija et al., 2009; Jaitz et al., 2010).

Anthocyanin concentrations are typical of the grape cultivar from which the wine is produced (Rossouw \& Marais, 2004). The anthocyanin concentrations change during the ageing of wine, but the ratios of the anthocyanins in wines from different cultivars remain distinctive (Ribéreau-Gayon et al., 2006).

Quantification of red wine polyphenols has been the focus of a number of studies in view of their contribution to wine sensory properties such as colour, flavour, astringency and bitterness, as well as their role in the differentiation of grape cultivars (Schwarz et al., 2003; Fang et al., 2008; Rastija et al., 2009; Jaitz et al., 2010). Polyphenols also contribute to the determination of the authenticity of wine based on cultivar, vintage and origin (Otteneder et al., 2004; Von Baer et al., 2005; Von Baer et al., 2008). Additionally, polyphenols are used for the prediction of the sensory properties and stability of wine (Arvanitoyannis et al., 1999).

*Corresponding author: E-mail: minnaarp@arc.agric.za

Aknowledgements: The authors would like to thank the ARC for funding the project, Elsabé Ferreira, manager of the South African National Wine Show Association for donating the wines and The Fruit, Vine and Wine Institute of the Agricultural Research Council for their support. 
Polyphenols are also used as markers for wine ageing (Fang et al., 2008).

In South Africa, limited research has been conducted on the polyphenol profiles of young wines (wines of current vintage after alcoholic and malolactic fermentation) and market-ready wines (commercially ready wines). Polyphenol compositional data of South African dry red table wines, particularly Cabernet Sauvignon, Pinotage and Shiraz are at present insufficient. Goldberg et al. (1998a, b) only quantified two flavanols and one flavonol and one phenolic acid in wine samples originating from different countries, including wine samples from South Africa. De Villiers et al. (2005) quantified a small number of flavanols and flavonols in red and white commercial wines. Rossouw \& Marias (2004) quantified polyphenols in young and market-ready wines. However, distinction between young and marketready wine polyphenol data was not reported.

The aim of this investigation was to quantify the concentration differences of flavonol, flavanol, anthocyanins and phenolic acids in young and market-ready Cabernet Sauvignon, Pinotage and Shiraz wines and to determine how useful certain variables (Polyphenols) are to differentiate among the grape cultivars of Pinotage, Shiraz and Cabernet Sauvignon using discriminant analysis. Additionally, discriminant analysis was also applied to distinguish among young and market-ready Pinotage, Shiraz and Cabernet Sauvignon wines.

\section{MATERIALS AND METHODS \\ Reagents}

The solvents used were of analytical grade and purchased from Merck ${ }^{\circledR}$, South Africa. De-ionised water was supplied through a Modulab ${ }^{\circledR}$ water purification system, supplied by Separations ${ }^{\circledR}$, South Africa. Flavonoid and non-flavonoid standards were obtained from Extrasynthèse ${ }^{\circledR}$, Genay, France. Stock solutions of gallic acid, catechin, caffeic acid, delphinidin glucoside, quercetin glucoside and rutin standards were prepared in an acidified methanol solution, i.e., methanol/water/formic acid with 50:48.5:4.5 ratios. Calibration curves were obtained from duplicate injections of four concentrations for each standard. The remaining analytes were interpolated in the calibration curves.

\section{Wine samples}

Three hundred and seventy-one young wines (unwooded) and 385 market-ready wines (wood-matured) produced from Cabernet Sauvignon, Pinotage and Shiraz grapes originating from the Western Cape Province in South Africa were collected at the annual Young Wine and Veritas Wine Shows held in Paarl, South Africa during 2002 and 2003. The collected market-ready wines represented 134 Cabernet Sauvignon samples, 125 Pinotage samples and 126 Shiraz samples. The young wines consisted of 123 Cabernet Sauvignon samples, 131 Pinotage samples and 117 Shiraz samples. Wine samples were not categorised according to origin. All young wines were confirmed on the bottle label as single cultivar wines. Market-ready wines were viewed as blends of up to $15 \%$ of another grape cultivar, however, the samples were analysed as the grape cultivar stated on the label. All wine samples were dry red table wines with a residual sugar of $<5 \mathrm{~g} / \mathrm{L}$. Wine samples were stored at $0^{\circ} \mathrm{C}$ within 12 hours after collection and remained at $0^{\circ} \mathrm{C}$ until required for analysis.

\section{Sample preparation}

Wine samples were analysed without any pre-treatment, except filtering through a $33 \mathrm{~mm}$, non-sterile, $0.45 \mu \mathrm{m}$ syringe filter (Millex Syringe Filter Units) supplied by Microsep $^{\circledR}$, South Africa.

\section{High-performance Liquid Chromatography Diode- Array Detection (HPLC-DAD)}

High-performance liquid chromatographic determination of phenolic acids, flavonols, flavanols and anthocyanins was performed using a Thermo Separations products ${ }^{\circledR}$ HPLC, supplied by Spectra System Separation Products. The HPLC was equipped with an auto sampler, injecting a $20 \mu \mathrm{l}$ sample. Detection was achieved by means of photodiode array. ChromQuest $^{\mathbb{R}}$ software was utilised for data acquisition and calibrations. Separation was performed at ca. $22^{\circ} \mathrm{C}$, using a polymer reverse phase analytical column (PLRP-S 100 $\AA, 5 \mu \mathrm{m}, 250 \times 6.6 \mathrm{~mm}$ ) with polystyrene divinylbenzene as stationary phase. The column was supplied by Polymer Laboratories $^{\circledR}$, USA. Gradient elution with two solvents was used: solvent A consisted of phosphoric acid $(\mathrm{pH}=3.0)$ with water (15:985 v/v) and solvent B was phosphoric acid $(\mathrm{pH}=3.0)$ with acetonitrile and water $(15: 800: 185 \mathrm{v} / \mathrm{v})$. The following gradient of eluent was used: $6 \% \mathrm{~B}$ initially, $6-62 \%$ B, 0-86 min.; 62-6\% B, 86-90 min. Reverting to the starting conditions, $20 \mathrm{~min}$ prior to the injection of the next sample resulted in the equilibration of the system. The flow rate was $1 \mathrm{~mL} / \mathrm{min}$. Ultra-violet visible spectra were recorded for all peaks. The analytical method is based on the method described by Waterhouse et al. (1999) for wine polyphenol separation and quantification. Anthocyanins were detected at $520 \mathrm{~nm}$ using malvidin-3-O-glucoside as external standard. Hydroxycinnamic acids were detected at $316 \mathrm{~nm}$ using caffeic acid as external standards. Flavonols were detected at $360 \mathrm{~nm}$ using rutin and flavanols at $280 \mathrm{~nm}$ using $(+)$-catechin as external standards. The identification of these compounds was confirmed by their relative retention times and UV-visible absorption characteristics, using the method of Waterhouse et al. (1999) as reference. This method is endorsed by the Office International de la Vigne et $d u$ Vin (OIV, Resolution Oeno 22/2003). Duplicate samples for each of the grape cultivars, young and market-ready wines were taken from two separate bottles of each wine. Samples were separately analysed. Results were recorded from calibration curves and spectral data and expressed as milligrams per litre. Average values were used for statistical analyses.

\section{Statistical analysis}

Discriminant analysis can be used to analyse data from all the different individual polyphenols to determine whether discrimination among grape cultivars and young and marketready wines is possible. Correlation and multiple linear regressions was performed using the $\mathrm{XLSTAT}^{\circledR}$ version 8.03 (2007) programme. Multiple linear regression analysis was carried out in a forward stepwise manner to select suitable 
variables (polyphenols) in the model. Grape cultivars were considered as classification variables and polyphenols were the $\mathrm{x}$-variables (independent variables) (Table 1). Only the variables showing significant correlation $(p<0.05)$ were included in the analysis. All data were processed using XLSTAT $^{\circledR}$ version 8.03 (2007).

\section{Stepwise discriminant analysis}

Stepwise discriminant analysis (SDA) is a preliminary analysis and the resulting subset of variables was used in canonical discriminant analysis (CDA). Stepwise discriminant analysis was used to select a sub-set of variables from the original thirty-three variables. The subset contained those polyphenol compounds which best differentiate or discriminate among grape cultivars and young and marketready wines.

\section{Canonical discriminant analysis}

The primary purpose of CDA is to identify natural groupings or patterns among the data. Linear functions of the variables (discriminant functions) are used to describe the differences among two or more groups. These linear combinations known as canonical variables are plotted on an axis to obtain a two-dimensional graph, which depicts the discrimination of grape cultivars among classes. The first two canonical variables (Can. 1 and Can. 2) are plotted against each other, since they account for the most significant discrimination of grape cultivars among classes.

The concentrations of the polyphenol compounds are subjected to canonical discriminant analysis to evaluate the potential of the polyphenol pattern for use in discrimination and to classify the samples according to grape cultivar, young wines and market-ready wines. If discrimination among grape cultivars of young and market-ready wines is possible, CDA will classify the data into the correct groups and consequently attain its classification accuracy.

\section{RESULTS AND DISCUSSION}

\section{Polyphenol composition of wine}

Thirty-three individual polyphenol compounds were initially quantified in the young and market-ready wine samples (Table 1).

Table 1 shows the mean concentrations of polyphenols determined in young and market-ready Pinotage, Cabernet
Sauvignon and Shiraz. The results obtained from the analysed wine samples confirm variations in the individual polyphenol concentrations of the wines made from different grape cultivars as well as differences between young and market-ready wines. Some of the most striking concentration differences were higher mean levels of caftaric acid, caffeic acid and quercetin aglycone in young and market-ready wines and differences between grape cultivars. Young wines contained higher levels of glycosidic anthocyanins and acetylated anthocyanins compared to market-ready wines.

\section{Classification of Wine \\ Young wines}

Concentration differences were implemented as a means to classify young Cabernet Sauvignon, Pinotage and Shiraz wines. Canonical discriminant analysis was applied to the data in an attempt to differentiate among grape cultivars based on their polyphenol compound composition.

Canonical discriminant analysis was based on significant $F$-values. According to Rencher (2002), standardised canonical variable coefficients with a value of \pm 0.5 and higher correlation are variables that contribute to the classification of grape cultivars. These coefficients were considered to be dominant variables. The canonical function loadings of the discriminant analysis (Table 2) indicated that concentrations of peonidin glucoside acetate, petunidin glucoside $p$-coumarate, delphinidin glucoside $p$-coumarate, malvidin glucoside $p$-coumarate, quercetin-3 glucoside, quercetin aglycone and isorhamnetin were most important in discriminating among cultivars using function 1 (Canonical component 1). The concentrations of catechin, tryptophol, epigallocatechin gallate and caftaric were most important to distinguish grape cultivars using function 2 (Canonical component 2). Function 1 was the most effective function in discriminating young Pinotage and Cabernet Sauvignon wines but not Shiraz wines (Fig. 1). Only one sample declared as Pinotage falls in the group of Cabernet Sauvignon. Class centroids (ellipsoids) for the different grape cultivar (including market-ready wines), were identified with different colours of the same shape. The graphic representation shown in Fig. 2 indicates a pattern of point distribution which distinguishes three groups corresponding to grape cultivar.

\section{TABLE 1}

Minimum, maximum and mean concentrations (mg/L) of individual phenolic compounds quantified in young and market-ready Cabernet Sauvignon, Shiraz and Pinotage wines.

\begin{tabular}{lccc|ccc}
\hline \multirow{3}{*}{ Polyphenols } & \multicolumn{3}{c|}{ Young wines } & \multicolumn{3}{c}{ Market-ready wines } \\
\cline { 2 - 7 } & $\begin{array}{c}\mathbf{1} \text { Cab. S } \\
(\mathbf{n}=\mathbf{1 2 3})^{\mathbf{2}}\end{array}$ & $\begin{array}{c}\text { Shiraz } \\
(\mathbf{n}=\mathbf{1 1 7})\end{array}$ & $\begin{array}{c}\text { Pinotage } \\
(\mathbf{n}=\mathbf{1 3 1})\end{array}$ & $\begin{array}{c}\text { Cab. S } \\
(\mathbf{n}=\mathbf{1 3 4})\end{array}$ & $\begin{array}{c}\text { Shiraz } \\
(\mathbf{n}=\mathbf{1 2 6})\end{array}$ & $\begin{array}{c}\text { Pinotage } \\
(\mathbf{n}=\mathbf{1 2 5})\end{array}$ \\
\cline { 2 - 7 } & Min-Max & Min-Max & Min-Max & Min-Max & Min-Max & Min-Max \\
& Mean $(\mathrm{SD})^{3}$ & Mean (SD) & Mean $(\mathrm{SD})$ & Mean $(\mathrm{SD})$ & Mean $(\mathrm{SD})$ & Mean $(\mathrm{SD})$ \\
\hline \multirow{2}{*}{ Gallic Acid } & $16.14-50.72$ & $7.63-51.32$ & $7.00-51.87$ & $14.21-83.55$ & $16.79-56.06$ & $8.09-61.90$ \\
& $30.24( \pm 8.55)$ & $28.68( \pm 9.08)$ & $27.00( \pm 9.87)$ & $43.44( \pm 14.07)$ & $33.62( \pm 10.06)$ & $27.79( \pm 11.09)$ \\
Protocatechuic acid & $0.15-2.49$ & $0.20-4.22$ & $0.19-1.29$ & $0.30-3.28$ & $0.29-5.46$ & $0.06-4.81$ \\
& $0.74( \pm 0.48)$ & $0.98( \pm 0.83)$ & $0.48( \pm 0.26)$ & $1.24( \pm 0.70)$ & $1.89( \pm 1.55)$ & $1.68( \pm 1.27)$ \\
Procyanidin B1 & $12.80-52.28$ & $24.37-53.56$ & $7.14-63.77$ & $17.91-80.16$ & $7.95-32.58$ & $12.69-58.35$ \\
& $25.25( \pm 10.04)$ & $38.72( \pm 7.01)$ & $37.02( \pm 15.53)$ & $43.06( \pm 17.24)$ & $23.32( \pm 6.17)$ & $35.84( \pm 8.68)$ \\
Catechin & $26.70-117.30$ & $27.94-71.65$ & $24.28-64.15$ & $24.32-66.63$ & $24.67-100.77$ & $12.75-52.88$ \\
& $60.11( \pm 20.97)$ & $48.08( \pm 11.69)$ & $42.84( \pm 9.69)$ & $43.46( \pm 10.24)$ & $64.20( \pm 19.69)$ & $30.70( \pm 7.97)$ \\
\hline
\end{tabular}


TABLE 1 (CONTINUED)

Minimum, maximum and mean concentrations $(\mathrm{mg} / \mathrm{L})$ of individual phenolic compounds quantified in young and market-ready Cabernet Sauvignon, Shiraz and Pinotage wines.

\begin{tabular}{|c|c|c|c|c|c|c|}
\hline \multirow[b]{2}{*}{ Polyphenols } & \multicolumn{3}{|c|}{ Young wines } & \multicolumn{3}{|c|}{ Market-ready wines } \\
\hline & $\begin{array}{c}{ }^{1} \text { Cab. S } \\
(\mathbf{n}=\mathbf{1 2 3})^{2}\end{array}$ & $\begin{array}{c}\text { Shiraz } \\
(\mathrm{n}=117)\end{array}$ & $\begin{array}{l}\text { Pinotage } \\
(\mathrm{n}=131)\end{array}$ & $\begin{array}{c}\text { Cab. S } \\
(n=134)\end{array}$ & $\begin{array}{c}\text { Shiraz } \\
(\mathrm{n}=126)\end{array}$ & $\begin{array}{l}\text { Pinotage } \\
(n=125)\end{array}$ \\
\hline & $\begin{array}{c}\text { Min-Max } \\
\text { Mean }(\mathrm{SD})^{3}\end{array}$ & $\begin{array}{l}\text { Min-Max } \\
\text { Mean (SD) }\end{array}$ & $\begin{array}{l}\text { Min-Max } \\
\text { Mean (SD) }\end{array}$ & $\begin{array}{l}\text { Min-Max } \\
\text { Mean (SD) }\end{array}$ & $\begin{array}{l}\text { Min-Max } \\
\text { Mean (SD) }\end{array}$ & $\begin{array}{l}\text { Min-Max } \\
\text { Mean (SD) }\end{array}$ \\
\hline Epicatechin & $\begin{array}{c}15.30-70.57 \\
38.93( \pm 12.96)\end{array}$ & $\begin{array}{c}15.46-73.62 \\
41.08( \pm 14.67)\end{array}$ & $\begin{array}{c}14.60-55.88 \\
33.99( \pm 10.84)\end{array}$ & $\begin{array}{c}12.52-69.84 \\
35.69( \pm 15.47)\end{array}$ & $\begin{array}{c}12.49-69.45 \\
25.54( \pm 13.51)\end{array}$ & $\begin{array}{c}11.69-50.06 \\
21.45( \pm 10.15)\end{array}$ \\
\hline Procyanidin B2 & $\begin{array}{c}21.62-47.80 \\
32.47( \pm 8.88)\end{array}$ & $\begin{array}{c}13.66-46.00 \\
27.90( \pm 7.85)\end{array}$ & $\begin{array}{c}18.10-40.75 \\
28.28( \pm 7.43)\end{array}$ & $\begin{array}{c}9.13-60.02 \\
23.49( \pm 12.44)\end{array}$ & $\begin{array}{c}11.08-58.82 \\
27.83( \pm 15.12)\end{array}$ & $\begin{array}{c}8.87-57.12 \\
23.23( \pm 14.19)\end{array}$ \\
\hline $\begin{array}{l}\text { Epigallocatechin } \\
\text { gallate }\end{array}$ & $\begin{array}{c}5.16-19.66 \\
10.13( \pm 3.51)\end{array}$ & $\begin{array}{c}6.00-43.15 \\
21.18( \pm 10.43)\end{array}$ & $\begin{array}{c}9.52-72.49 \\
28.24( \pm 13.52)\end{array}$ & $\begin{array}{c}4.61-19.56 \\
9.84( \pm 4.09)\end{array}$ & $\begin{array}{c}4.64-15.93 \\
9.40( \pm 2.79)\end{array}$ & $\begin{array}{c}2.61-34.63 \\
11.76( \pm 6.63)\end{array}$ \\
\hline Epicatechin gallate & $\begin{array}{c}5.10-20.93 \\
9.52( \pm 3.46)\end{array}$ & $\begin{array}{c}3.72-20.45 \\
7.03( \pm 4.30)\end{array}$ & $\begin{array}{c}4.46-13.63 \\
7.83( \pm 2.63)\end{array}$ & $\begin{array}{l}4.13-10.61 \\
6.76( \pm 1.66)\end{array}$ & $\begin{array}{c}3.66-10.79 \\
5.55( \pm 1.81)\end{array}$ & $\begin{array}{c}0.51-13.57 \\
5.45( \pm 3.80)\end{array}$ \\
\hline Tryptophol & $\begin{array}{c}1.54-8.67 \\
5.64( \pm 1.84)\end{array}$ & $\begin{array}{c}0.90-9.50 \\
4.20( \pm 2.18)\end{array}$ & $\begin{array}{c}0.37-5.87 \\
2.34( \pm 1.20)\end{array}$ & $\begin{array}{c}1.18-4.23 \\
2.66( \pm 0.922)\end{array}$ & $\begin{array}{c}0.40-4.67 \\
2.03( \pm 1.08)\end{array}$ & $\begin{array}{c}0.34-4.17 \\
1.73( \pm 0.97)\end{array}$ \\
\hline Polymeric phenols & $\begin{array}{c}179.32-500.62 \\
322.27( \pm 85.40)\end{array}$ & $\begin{array}{c}221.09-729.41 \\
404.71( \pm 117.39)\end{array}$ & $\begin{array}{c}118.59-700.65 \\
326.08( \pm 139.69)\end{array}$ & $\begin{array}{c}252.86-587.27 \\
377.02( \pm 90.68)\end{array}$ & $\begin{array}{c}220.89-535.15 \\
346.43( \pm 78.14)\end{array}$ & $\begin{array}{c}185.58-513.42 \\
316.67( \pm 87.84)\end{array}$ \\
\hline $\begin{array}{l}\text { Quercetin-3 } \\
\text { glucoside }\end{array}$ & $\begin{array}{c}2.77-43.55 \\
10.38( \pm 8.35)\end{array}$ & $\begin{array}{c}3.59-81.00 \\
21.63( \pm 17.31)\end{array}$ & $\begin{array}{c}2.9431 .06 \\
13.18( \pm 7.4)\end{array}$ & $\begin{array}{c}2.23-29.33 \\
7.45( \pm 5.37)\end{array}$ & $\begin{array}{c}2.39-29.66 \\
10.99( \pm 6.39)\end{array}$ & $\begin{array}{c}1.93-41.58 \\
12.95( \pm 9.64)\end{array}$ \\
\hline Quercetin aglycone & $\begin{array}{c}10.29-56.25 \\
29.84( \pm 11.24)\end{array}$ & $\begin{array}{c}5.10-84.65 \\
40.08( \pm 18.55)\end{array}$ & $\begin{array}{c}3.27-37.11 \\
15.37( \pm 7.13)\end{array}$ & $\begin{array}{c}3.71-32.24 \\
14.47( \pm 8.03)\end{array}$ & $\begin{array}{c}5.06-44.47 \\
17.11( \pm 8.60)\end{array}$ & $\begin{array}{c}6.11-31.58 \\
13.23( \pm 5.66)\end{array}$ \\
\hline Kaempferol & $\begin{array}{c}1.69-8.50 \\
4.21( \pm 1.73)\end{array}$ & $\begin{array}{c}1.26-9.50 \\
4.03( \pm 1.90)\end{array}$ & $\begin{array}{c}1.25-5.82 \\
3.06( \pm 1.23)\end{array}$ & $\begin{array}{c}1.21-4.43 \\
2.26( \pm 0.81)\end{array}$ & $\begin{array}{c}1.20-6.56 \\
2.53( \pm 1.42)\end{array}$ & $\begin{array}{c}0.61-3.17 \\
1.47( \pm 0.56)\end{array}$ \\
\hline Isorhamnetin & $\begin{array}{c}1.68-8.49 \\
3.79( \pm 1.50)\end{array}$ & $\begin{array}{c}2.38-16.90 \\
7.68( \pm 3.15)\end{array}$ & $\begin{array}{c}1.20-4.04 \\
2.02( \pm 0.61)\end{array}$ & $\begin{array}{c}1.36-5.29 \\
2.64( \pm 0.98)\end{array}$ & $\begin{array}{c}1.64-8.78 \\
3.68( \pm 1.87)\end{array}$ & $\begin{array}{c}0.64-3.29 \\
1.37( \pm 0.55)\end{array}$ \\
\hline Caftaric acid & $\begin{array}{c}2.65-47.65 \\
21.66( \pm 11.55)\end{array}$ & $\begin{array}{c}1.47-58.15 \\
24.35( \pm 13.84)\end{array}$ & $\begin{array}{c}5.89-105.48 \\
51.01( \pm 28.87)\end{array}$ & $\begin{array}{c}3.04-50.53 \\
21.84( \pm 14.43)\end{array}$ & $\begin{array}{c}4.46-36.83 \\
20.80( \pm 9.48)\end{array}$ & $\begin{array}{c}10.18-60.85 \\
36.27( \pm 16.53)\end{array}$ \\
\hline Caffeic acid & $\begin{array}{c}3.49-42.97 \\
13.06( \pm 9.14)\end{array}$ & $\begin{array}{c}4.08-41.31 \\
15.29( \pm 9.71)\end{array}$ & $\begin{array}{c}4.95-89.18 \\
36.90( \pm 27.25)\end{array}$ & $\begin{array}{c}6.18-48.96 \\
23.98( \pm 10.16)\end{array}$ & $\begin{array}{c}6.29-41.79 \\
20.47( \pm 10.26)\end{array}$ & $\begin{array}{c}9.11-59.65 \\
31.23( \pm 13.30)\end{array}$ \\
\hline Coutaric acid & $\begin{array}{c}1.88-27.52 \\
10.72( \pm 6.26)\end{array}$ & $\begin{array}{c}2.40-29.81 \\
15.18( \pm 7.68)\end{array}$ & $\begin{array}{c}0.65-57.02 \\
14.51( \pm 11.53)\end{array}$ & $\begin{array}{c}2.85-27.06 \\
11.31( \pm 6.15)\end{array}$ & $\begin{array}{c}2.38-35.45 \\
9.63( \pm 6.87)\end{array}$ & $\begin{array}{c}1.79-25.09 \\
10.19( \pm 6.21)\end{array}$ \\
\hline$p$-Coumaric acid & $\begin{array}{c}1.73-19.69 \\
6.31( \pm 4.58)\end{array}$ & $\begin{array}{c}1.71-21.56 \\
7.79( \pm 5.29)\end{array}$ & $\begin{array}{c}1.72-22.05 \\
9.57( \pm 6.62)\end{array}$ & $\begin{array}{c}1.66-17.54 \\
7.61( \pm 3.69)\end{array}$ & $\begin{array}{c}1.50-12.39 \\
5.55( \pm 3.21)\end{array}$ & $\begin{array}{c}2.40-27.12 \\
9.89( \pm 5.98)\end{array}$ \\
\hline $\begin{array}{l}\text { Delphinidin } \\
\text { 3-O-glucoside }\end{array}$ & $\begin{array}{c}6.53-38.45 \\
18.81( \pm 8.86)\end{array}$ & $\begin{array}{c}4.04-20.66 \\
10.69( \pm 4.23)\end{array}$ & $\begin{array}{c}4.65-30.52 \\
14.49( \pm 5.88)\end{array}$ & $\begin{array}{c}1.07-14.23 \\
5.15( \pm 3.25)\end{array}$ & $\begin{array}{c}1.72-9.84 \\
4.45( \pm 1.95)\end{array}$ & $\begin{array}{c}1.64-14.11 \\
5.58( \pm 2.65)\end{array}$ \\
\hline Cyanidin & $0.92-4.68$ & $0.62-2.73$ & $0.82-3.33$ & $0.61-1.85$ & $0.61-1.37$ & $0.58-1.50$ \\
\hline 3-O-glucoside & $1.97( \pm 0.96)$ & $1.31( \pm 0.41)$ & $1.54( \pm 0.55)$ & $0.94( \pm 0.28)$ & $0.86( \pm 0.20)$ & $0.91( \pm 0.21)$ \\
\hline Petunidin & $4.75-33.41$ & $6.99-32.07$ & $8.02-40.52$ & $0.86-16.50$ & $1.03-12.99$ & $1.09-20.77$ \\
\hline 3-O-glucoside & $18.52( \pm 6.68)$ & $17.88( \pm 6.37)$ & $21.88( \pm 6.97)$ & $4.53( \pm 3.27)$ & $5.46( \pm 3.11)$ & $6.16( \pm 4.18)$ \\
\hline $\begin{array}{l}\text { Peonidin } \\
\text { 3-O-glucoside }\end{array}$ & $\begin{array}{c}3.13-19.37 \\
9.51( \pm 4.11)\end{array}$ & $\begin{array}{c}5.82-23.26 \\
12.15( \pm 3.84)\end{array}$ & $\begin{array}{c}2.41-20.00 \\
9.26( \pm 3.88)\end{array}$ & $\begin{array}{c}0.75-7.22 \\
2.37( \pm 1.58)\end{array}$ & $\begin{array}{c}0.87-8.06 \\
3.85( \pm 1.70)\end{array}$ & $\begin{array}{c}0.79-9.43 \\
3.03( \pm 1.90)\end{array}$ \\
\hline $\begin{array}{l}\text { Malvidin } \\
\text { 3-O-glucoside }\end{array}$ & $\begin{array}{c}95.52-234.37 \\
158.93( \pm 32.65)\end{array}$ & $\begin{array}{c}76.54-232.78 \\
150.05( \pm 36.82)\end{array}$ & $\begin{array}{c}95.14-269.18 \\
168.30( \pm 34.92)\end{array}$ & $\begin{array}{c}10.71-174.49 \\
44.29( \pm 33.06)\end{array}$ & $\begin{array}{c}18.85-106.45 \\
50.48( \pm 23.38)\end{array}$ & $\begin{array}{c}21.36-121.71 \\
56.67( \pm 23.97)\end{array}$ \\
\hline $\begin{array}{l}\text { Delphinidin } \\
\text { glucoside acetate }\end{array}$ & $\begin{array}{c}1.62-15.61 \\
6.86( \pm 3.11)\end{array}$ & $\begin{array}{c}0.62-9.52 \\
4.21( \pm 2.09)\end{array}$ & $\begin{array}{c}3.01-10.41 \\
6.45( \pm 1.88)\end{array}$ & $\begin{array}{c}0.60-5.24 \\
2.15( \pm 1.21)\end{array}$ & $\begin{array}{c}0.66-6.44 \\
1.85( \pm 1.20)\end{array}$ & $\begin{array}{c}0.54-3.33 \\
1.37( \pm 0.59)\end{array}$ \\
\hline Vitisin A & $\begin{array}{c}1.17-6.87 \\
3.83( \pm 1.11)\end{array}$ & $\begin{array}{c}1.48-5.95 \\
3.11( \pm 0.89)\end{array}$ & $\begin{array}{c}1.30-6.56 \\
3.11( \pm 1.17)\end{array}$ & $\begin{array}{c}1.02-7.54 \\
2.57( \pm 1.53)\end{array}$ & $\begin{array}{c}0.86-5.30 \\
2.44( \pm 1.19)\end{array}$ & $\begin{array}{c}1.36-6.47 \\
3.07( \pm 1.15)\end{array}$ \\
\hline $\begin{array}{l}\text { Petunidin glucoside } \\
\text { acetate }\end{array}$ & $\begin{array}{c}1.91-15.86 \\
7.58( \pm 2.47)\end{array}$ & $\begin{array}{c}2.13-10.20 \\
6.14( \pm 1.65)\end{array}$ & $\begin{array}{c}2.04-10.39 \\
6.47( \pm 1.96)\end{array}$ & $\begin{array}{c}0.99-7.18 \\
2.94( \pm 1.26)\end{array}$ & $\begin{array}{c}0.85-5.25 \\
2.35( \pm 1.11)\end{array}$ & $\begin{array}{c}0.58-4.32 \\
2.20( \pm 0.85)\end{array}$ \\
\hline Acetylated vitisin A & $\begin{array}{c}0.58-3.14 \\
0.96( \pm 0.61)\end{array}$ & $\begin{array}{c}0.56-5.73 \\
1.47( \pm 1.16)\end{array}$ & $\begin{array}{c}0.57-4.16 \\
1.50( \pm 1.11)\end{array}$ & $\begin{array}{c}0.57-1.03 \\
0.67( \pm 0.10)\end{array}$ & $\begin{array}{c}0.85-2.71 \\
1.71( \pm 0.48)\end{array}$ & $\begin{array}{c}0.50-2.56 \\
1.35( \pm 0.59)\end{array}$ \\
\hline $\begin{array}{l}\text { Peonidin glucoside } \\
\text { acetate }\end{array}$ & $\begin{array}{c}1.48-8.88 \\
5.11( \pm 1.79)\end{array}$ & $\begin{array}{c}1.22-19.26 \\
9.17( \pm 4.40)\end{array}$ & $\begin{array}{c}1.78-9.88 \\
5.41( \pm 1.62)\end{array}$ & $\begin{array}{c}0.59-4.60 \\
1.43( \pm 0.91)\end{array}$ & $\begin{array}{c}0.61-5.67 \\
2.21( \pm 1.28)\end{array}$ & $\begin{array}{c}0.63-5.37 \\
1.75( \pm 0.99)\end{array}$ \\
\hline $\begin{array}{l}\text { Malvidin glucoside } \\
\text { acetate }\end{array}$ & $\begin{array}{c}23.82-117.94 \\
66.34( \pm 18.84)\end{array}$ & $\begin{array}{c}6.61-83.43 \\
49.40( \pm 17.70)\end{array}$ & $\begin{array}{c}11.85-75.11 \\
48.75( \pm 12.66)\end{array}$ & $\begin{array}{c}2.02-60.29 \\
15.04( \pm 13.70)\end{array}$ & $\begin{array}{c}1.03-38.30 \\
13.60( \pm 9.97)\end{array}$ & $\begin{array}{c}1.93-35.95 \\
14.41( \pm 8.62)\end{array}$ \\
\hline $\begin{array}{l}{ }^{4} \text { Delphi glucoside } \\
p \text {-coumarate }\end{array}$ & $\begin{array}{c}0.62-3.86 \\
1.84( \pm 0.69)\end{array}$ & $\begin{array}{c}1.17-5.46 \\
3.60( \pm 0.82)\end{array}$ & $\begin{array}{c}1.31-4.95 \\
2.32( \pm 0.67)\end{array}$ & $\begin{array}{c}0.60-2.31 \\
1.03( \pm 0.37)\end{array}$ & $\begin{array}{c}0.67-2.50 \\
1.31( \pm 0.45)\end{array}$ & $\begin{array}{c}0.57-3.88 \\
1.30( \pm 0.92)\end{array}$ \\
\hline $\begin{array}{l}{ }^{5} \text { Petuni glucoside } \\
p \text {-coumarate }\end{array}$ & $\begin{array}{c}1.39-5.43 \\
3.07( \pm 0.90)\end{array}$ & $\begin{array}{c}3.93-9.52 \\
6.87( \pm 1.48)\end{array}$ & $\begin{array}{c}1.69-5.73 \\
3.40( \pm 0.91)\end{array}$ & $\begin{array}{c}0.673 .79 \\
1.34( \pm 0.68)\end{array}$ & $\begin{array}{c}0.72-4.33 \\
1.82( \pm 0.85)\end{array}$ & $\begin{array}{c}0.65-3.47 \\
1.40( \pm 0.58)\end{array}$ \\
\hline
\end{tabular}


TABLE 1 (CONTINUED)

Minimum, maximum and mean concentrations (mg/L) of individual phenolic compounds quantified in young and market-ready Cabernet Sauvignon, Shiraz and Pinotage wines.

\begin{tabular}{|c|c|c|c|c|c|c|}
\hline \multirow[b]{2}{*}{ Polyphenols } & \multicolumn{3}{|c|}{ Young wines } & \multicolumn{3}{|c|}{ Market-ready wines } \\
\hline & $\begin{array}{c}{ }^{1} \text { Cab. S } \\
(\mathrm{n}=123)^{2}\end{array}$ & $\begin{array}{c}\text { Shiraz } \\
(\mathrm{n}=117)\end{array}$ & $\begin{array}{l}\text { Pinotage } \\
(\mathrm{n}=131)\end{array}$ & $\begin{array}{c}\text { Cab. S } \\
(n=134)\end{array}$ & $\begin{array}{c}\text { Shiraz } \\
(n=126)\end{array}$ & $\begin{array}{l}\text { Pinotage } \\
(\mathrm{n}=125)\end{array}$ \\
\hline & $\begin{array}{c}\text { Min-Max } \\
\text { Mean (SD) }\end{array}$ & $\begin{array}{l}\text { Min-Max } \\
\text { Mean (SD) }\end{array}$ & $\begin{array}{l}\text { Min-Max } \\
\text { Mean (SD) }\end{array}$ & $\begin{array}{l}\text { Min-Max } \\
\text { Mean (SD) }\end{array}$ & $\begin{array}{l}\text { Min-Max } \\
\text { Mean (SD) }\end{array}$ & $\begin{array}{l}\text { Min-Max } \\
\text { Mean (SD) }\end{array}$ \\
\hline Pinotin A & $\begin{array}{c}0.60-1.39 \\
0.83( \pm 0.19)\end{array}$ & $\begin{array}{c}0.72-1.89 \\
1.16( \pm 0.27)\end{array}$ & $\begin{array}{c}0.61-7.44 \\
1.29( \pm 1.29)\end{array}$ & $\begin{array}{c}0.65-2.60 \\
1.06( \pm 0.35)\end{array}$ & $\begin{array}{c}0.62-3.55 \\
1.15( \pm 0.56)\end{array}$ & $\begin{array}{c}0.65-9.18 \\
2.40( \pm 1.97)\end{array}$ \\
\hline $\begin{array}{l}{ }^{6} \text { Malvi. glucoside } \\
p \text {-coumarate }\end{array}$ & $\begin{array}{c}9.99-42.30 \\
19.53( \pm 7.26)\end{array}$ & $\begin{array}{c}19.90-59.52 \\
36.63( \pm 9.12)\end{array}$ & $\begin{array}{c}1.72-33.31 \\
16.45( \pm 7.59)\end{array}$ & $\begin{array}{c}0.84-20.68 \\
4.14( \pm 4.04)\end{array}$ & $\begin{array}{c}1.18-21.24 \\
6.68( \pm 4.18)\end{array}$ & $\begin{array}{c}1.19-15.08 \\
5.81( \pm 3.59)\end{array}$ \\
\hline Polymeric pigments & $\begin{array}{c}15.32-38.65 \\
24.48( \pm 6.21)\end{array}$ & $\begin{array}{c}15.52-47.63 \\
27.41( \pm 7.38)\end{array}$ & $\begin{array}{c}6.13-46.17 \\
21.39( \pm 7.67)\end{array}$ & $\begin{array}{c}14.40-52.74 \\
27.56( \pm 8.70)\end{array}$ & $\begin{array}{c}12.90-47.50 \\
24.87( \pm 8.06)\end{array}$ & $\begin{array}{c}8.37-47.52 \\
24.49( \pm 8.06)\end{array}$ \\
\hline $\begin{array}{l}{ }^{7} \text { Peoma glucoside } \\
\text { p-coumarate }\end{array}$ & $\begin{array}{c}7.99-46.00 \\
20.96( \pm 6.50)\end{array}$ & $\begin{array}{c}0.62-51.95 \\
24.52( \pm 14.65)\end{array}$ & $\begin{array}{c}7.21-57.24 \\
27.36( \pm 7.43)\end{array}$ & $\begin{array}{c}0.53-21.00 \\
3.71( \pm 4.16)\end{array}$ & $\begin{array}{c}0.50-9.44 \\
1.91( \pm 2.28)\end{array}$ & $\begin{array}{c}0.50-10.32 \\
2.13( \pm 2.11)\end{array}$ \\
\hline
\end{tabular}

${ }^{1} \mathrm{Cab} . \mathrm{S}=$ Cabernet Sauvignon; ${ }^{2} \mathrm{n}=$ Number of samples; ${ }^{3} \mathrm{SD}=$ Standard Deviation; ${ }^{4}$ Delphi $=$ Delphinidin; ${ }^{5}$ Petuni $=$ Petunidin $;{ }^{6}$ Malvi $=$ Malvidin $;{ }^{7}$ Peoma $=$ Peomalvidin

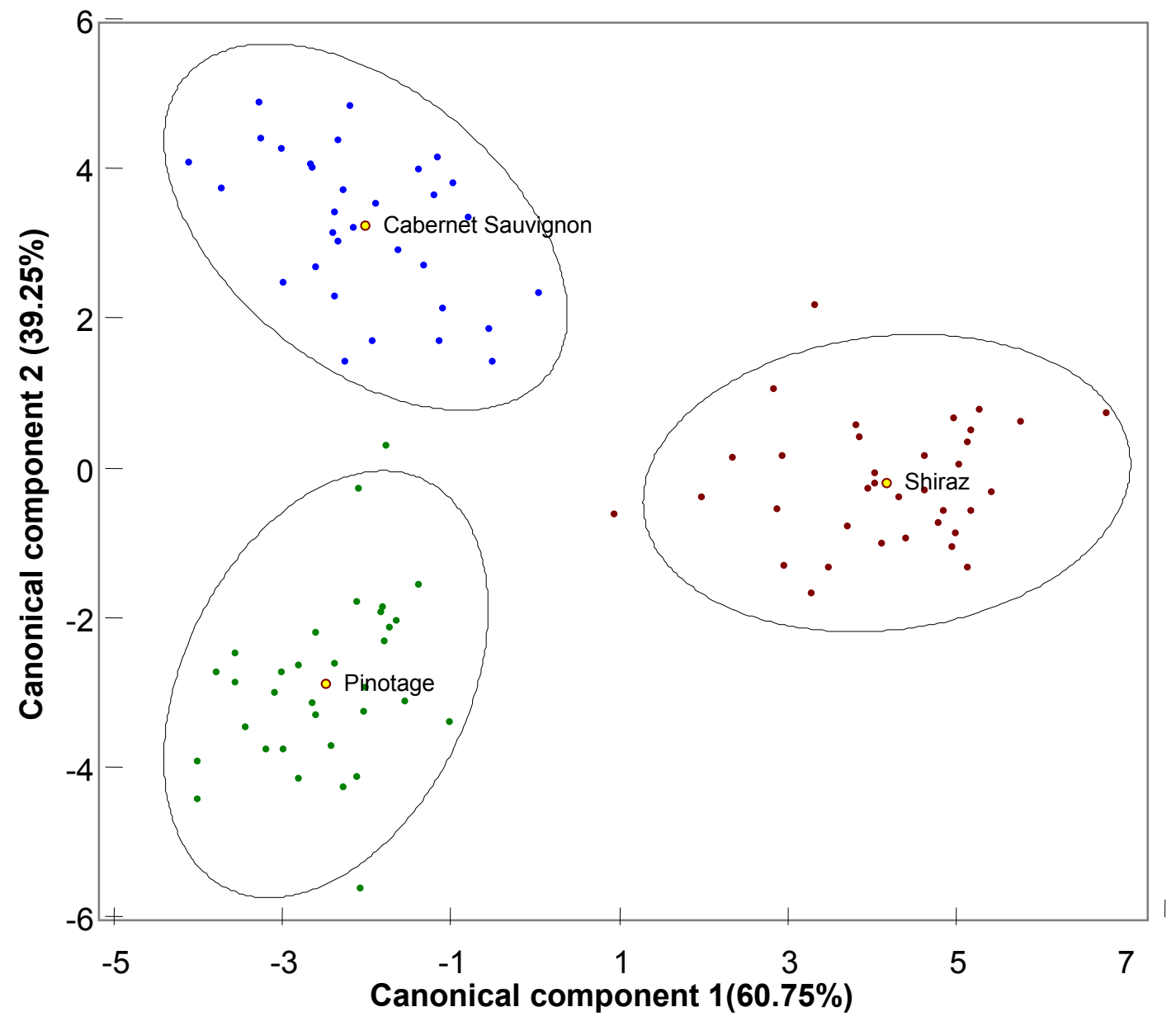

FIGURE 1

Plots of the first two canonical variables (Function 1 and Function 2) for young Cabernet Sauvignon, Pinotage and Shiraz wines showing differentiation based on peonidin glucoside acetate, petunidin glucoside $p$-coumarate, delphinidin glucoside $p$-coumarate, malvidin glucoside $p$-coumarate, catechin, quercetin-3 glucoside, tryptophol, quercetin aglycone, isorhamnetin, caftaric acid and epigallocatechin gallate. 
TABLE 2

Total sample standardised canonical coefficients for young Pinotage,Cabernet Sauvignon and Shiraz wines.

\begin{tabular}{|c|c|c|}
\hline Variable & Function 1 & Function 2 \\
\hline Gallic acid & -0.010 & 0.173 \\
\hline Delphinidin glucoside acetate & -0.488 & 0.113 \\
\hline Peonidin glucoside acetate & 0.600 & 0.020 \\
\hline Petunidin glucoside $p$-coumarate & 0.892 & -0.071 \\
\hline Malvidin-3-O-glucoside & -0.162 & 0.006 \\
\hline Petunidin glucoside acetate & -0.220 & 0.280 \\
\hline Malvidin glucoside acetate & -0.101 & 0.485 \\
\hline Petunidin-3-O-glucoside & -0.164 & -0.181 \\
\hline Delphinidin glucoside $p$-coumarate & 0.789 & -0.184 \\
\hline Malvidin glucoside $p$-coumarate & 0.817 & 0.044 \\
\hline Cyanidin 3-p-glucoside & -0.315 & 0.244 \\
\hline$p$-Coumaric acid & -0.052 & -0.188 \\
\hline Delphinidin 3-O-glucoside & -0.424 & 0.270 \\
\hline Peonidin 3-O-glucoside & 0.288 & 0.044 \\
\hline Epicatechin & 0.305 & 0.175 \\
\hline Catechin & -0.025 & 0.531 \\
\hline Pinotin A & 0.121 & -0.213 \\
\hline Polymeric pigments & 0.117 & 0.136 \\
\hline Quercetin-3 glucoside & 0.611 & -0.074 \\
\hline Tryptophol & 0.207 & 0.612 \\
\hline Protocatechuic acid & 0.360 & 0.154 \\
\hline Quercetin aglycone & 0.577 & 0.369 \\
\hline Epicatechin gallate & -0.124 & 0.164 \\
\hline Isorhamnetin & 0.779 & 0.249 \\
\hline Vitisin A & -0.115 & 0.285 \\
\hline Coutaric acid & 0.035 & -0.175 \\
\hline Polymeric phenols & 0.049 & -0.195 \\
\hline Kaempferol & 0.184 & 0.332 \\
\hline Caffeic acid & -0.271 & -0.478 \\
\hline Epigallocatechin gallate & -0.058 & -0.516 \\
\hline Procyanidin B1 & 0.233 & -0.263 \\
\hline Caftaric acid & -0.311 & -0.545 \\
\hline Acetylated vitisin A & -0.049 & -0.213 \\
\hline
\end{tabular}

\section{Market-ready wines}

Discriminant analysis could also distinguish market-ready wines. Table 3 lists the total sample standardised canonical coefficients for market-ready Pinotage, Cabernet Sauvignon and Shiraz wines.

The canonical function loadings of the discriminant analysis (Table 3 ) indicated that concentrations of petunidin glucoside $p$-coumarate, malvidin 3-O-glucoside, petunidin 3-O-glucoside, delphinidin glucoside $p$-coumarate, malvidin glucoside $p$-coumarate, quercetin-3 glucoside, quercetin aglycone, isorhamnetin and caftaric acid were most important in discriminating among cultivars using function 1 (Canonical component 1 ). The concentrations of petunidin glucoside $p$-coumarate, delphinidin glucoside $p$-coumarate, catechin, tryptophol, procyanidin B1, and epigallocatechin gallate were most important to distinguish grape cultivars using function 2 (Canonical component 2). Function 2 was the most effective function in discriminating market-ready Cabernet Sauvignon and Pinotage wines but not wines of Shiraz cultivar (Fig. 2). One sample declared as Pinotage falls in the group of Cabernet Sauvignon and two samples declared as Shiraz fall in the group of Cabernet Sauvignon. The graphic representation shown in Fig. 2 indicates a pattern of point distribution which distinguishes three groups corresponding to grape cultivar.

\section{Market-ready and young wines combined}

Discriminant analysis could also distinguish between marketready and young wines for each cultivar individually when 


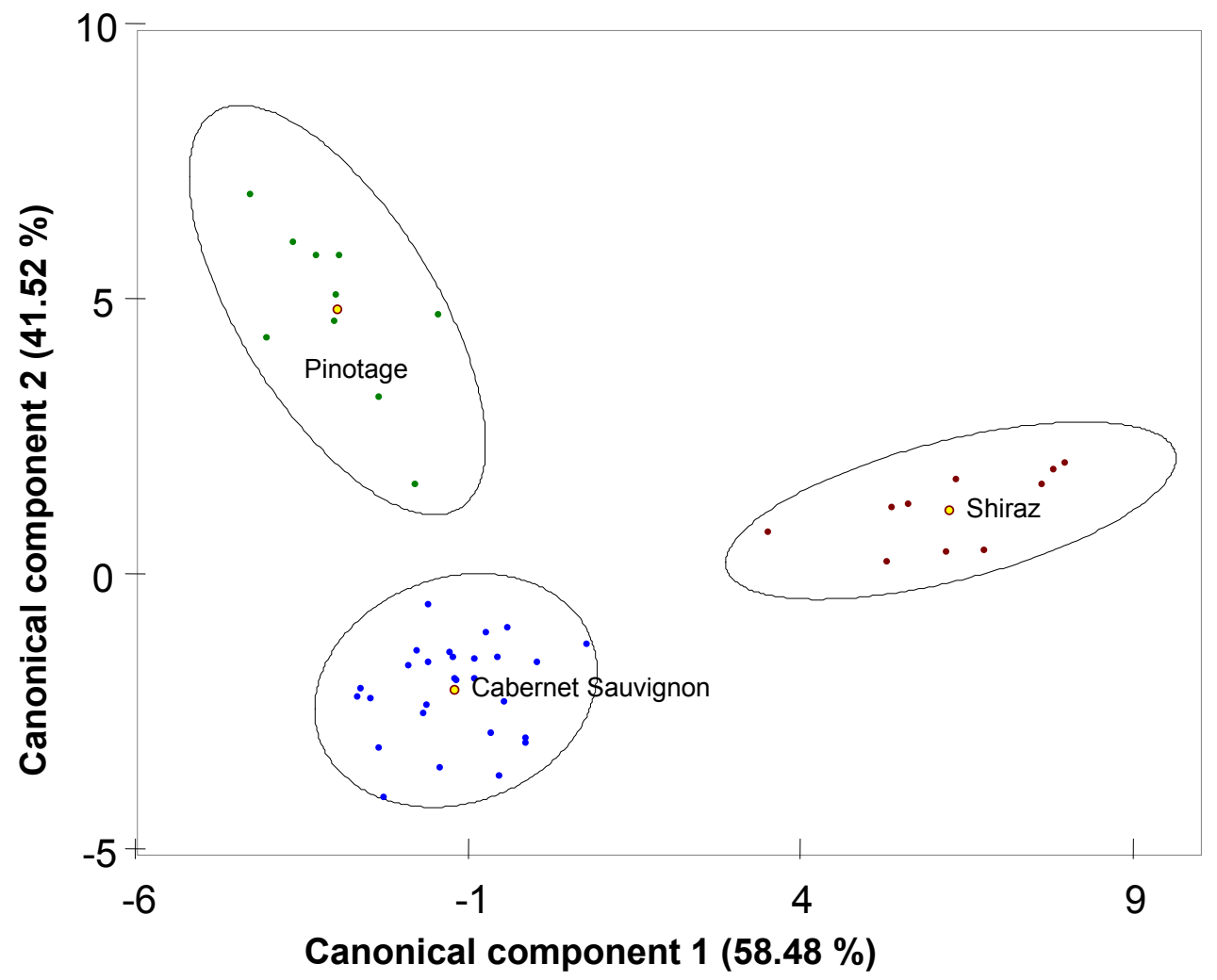

FIGURE 2

Plots of the first two canonical variables (Function 1 and Function 2) for market-ready Cabernet Sauvignon,

Pinotage and Shiraz wines showing differentiation based on petunidin glucoside $p$-coumarate, malvidin

3-O-glucoside, petunidin 3-O-glucoside, delphinidin glucoside $p$-coumarate, malvidin glucoside $p$-coumarate, catechin, quercetin-3 glucoside, tryptophol, quercetin aglycone, caftaric acid, epigallocatechin gallate, procyanidin B1and isorhamnetin

the polyphenol data is combined. The canonical coefficient function loadings indicated that for each cultivar, polyphenol compounds were selected to discriminate between marketready and young wines (Table 4 ).

The canonical function loadings of the discriminant analysis (Table 4) indicated that concentrations of peonidin glucoside acetate, petunidin glucoside $p$-coumarate, delphinidin glucoside $p$-coumarate, malvidin glucoside $p$-coumarate, peonidin 3-O-glucoside, isorhamnetin, quercetin aglycone and quercetin-3 glucoside were most important to discriminate between market-ready and young wines (based on cultivar) using function 1 (Canonical component 1). The concentrations of malvidin glucoside acetate, catechin and tryptophol were most important to discriminate between market-ready and young wines using function 2 (Canonical component 2). Function 1 was the most effective function in discriminating between young Cabernet Sauvignon and young Shiraz wines in comparison to the rest of the wines (Fig. 3). The graphical representation in Fig. 3 indicates a pattern of point distribution that corresponds with grape cultivar, young wines and market-ready wines.

Evaluation of the phenol-based differentiation of wines according to their grape cultivar revealed that two grape cultivars namely Shiraz and Pinotage delivered the most noticeable differences in polyphenol concentrations (Table 1). Flavonol concentrations were highest in young Shiraz and Cabernet Sauvignon wines, while they also showed higher average flavanol concentrations than other wines. Actually, the highest average concentrations of flavanols were quantified in young Shiraz wines. Young wines also exhibited a notably higher average concentration in anthocyanins compared to market-ready wines. However, anthocyanin levels within the three grape cultivars tested proved to be similar. These variations in phenolic composition reflect a natural qualitative difference among the analysed grape cultivars. Moreover, variation in berry maturation, berry size and berry weight at harvest and exposure of grapes to different climatic conditions during maturation contribute to differences among grape cultivars in phenolic profile (Ryan \& Revilla, 2003; Kallithraka et al., 2006; Cliff et al., 2007).

Anthocyanins were not selected as discriminant variables in market-ready wines, since a rapid decrease of anthocyanin concentration in market-ready wines occurs in the first few months during barrel and bottle ageing, 
TABLE 3

Total sample standardised canonical coefficients for market-ready Pinotage, Cabernet Sauvignon and Shiraz wines.

\begin{tabular}{|c|c|c|}
\hline Variable & Function 1 & Function 2 \\
\hline Gallic acid & 0.131 & -0.249 \\
\hline Delphinidin glucoside acetate & -0.447 & -0.167 \\
\hline Peonidin glucoside acetate & 0.484 & 0.308 \\
\hline Petunidin glucoside $p$-coumarate & 0.639 & 0.563 \\
\hline Malvidin 3-O-glucoside & -0.546 & -0.084 \\
\hline Petunidin glucoside acetate & -0.399 & -0.192 \\
\hline Malvidin glucoside acetate & -0.027 & -0.428 \\
\hline Petunidin 3-O-glucoside & -0.503 & -0.003 \\
\hline Delphinidin glucoside $p$-coumarate & 0.424 & 0.590 \\
\hline Malvidin glucoside $p$-coumarate & 0.630 & 0.282 \\
\hline Cyanidin 3-O-glucoside & -0.295 & -0.211 \\
\hline$p$-Coumaric acid & -0.059 & 0.201 \\
\hline Delphinidin 3-O-glucoside & -0.390 & -0.347 \\
\hline Peonidin 3-O-glucoside & 0.063 & 0.055 \\
\hline Epicatechin & 0.362 & -0.139 \\
\hline Catechin & 0.304 & -0.592 \\
\hline Pinotin A & 0.226 & 0.430 \\
\hline Polymeric pigments & 0.188 & -0.326 \\
\hline Quercetin-3 glucoside & 0.646 & 0.476 \\
\hline Tryptophol & 0.492 & -0.526 \\
\hline Protocatechuic acid & 0.484 & 0.230 \\
\hline Quercetin aglycone & 0.768 & -0.146 \\
\hline Epicatechin gallate & -0.263 & -0.271 \\
\hline Isorhamnetin & 0.840 & 0.064 \\
\hline Vitisin A & 0.046 & -0.331 \\
\hline Coutaric acid & -0.112 & 0.055 \\
\hline Polymeric phenols & 0.110 & 0.280 \\
\hline Kaempferol & 0.309 & -0.203 \\
\hline Caffeic acid & -0.406 & 0.362 \\
\hline Epigallocatechin gallate & -0.161 & 0.724 \\
\hline Procyanidin B1 & 0.057 & 0.565 \\
\hline Caftaric acid & -0.697 & 0.286 \\
\hline Acetylated vitisin A & -0.086 & 0.222 \\
\hline Gallic acid & 0.302 & 0.318 \\
\hline
\end{tabular}

reaching a value of around 0-50 $\mathrm{mg} / \mathrm{L}$ (Ribéreau-Gayon et al., 2006). In addition, the presence of ethyl alcohol in wines prevents co-pigmentation, which accounts for the decrease in anthocyanins. Acetylated and coumarylated anthocyanins disappear rapidly within a few months after the wine is processed because of the presence of ethyl alcohol, but anthocyanins, predominantly malvidin 3-O-glucoside, remain in the wine. The majority of these colour pigments combine and condense with the tannins and metals in wine to form stable procyanidin molecules and metal complexes. These complex anthocyanin molecules are responsible for the lasting and stable colour of red wine (Ribéreau-Gayon et al., 2006).

\section{CONCLUSIONS}

The data obtained from analysing young and market-ready Cabernet Sauvignon, Pinotage and Shiraz wines clearly indicate that certain grape cultivars are distinctive in terms of their polyphenol concentrations. This investigation revealed that flavonols, flavanols, and particularly anthocyanins, played an important part in the differentiation among grape cultivars in young wines and that flavonols and phenolic acids dominated the differentiation among market-ready wines.

The accumulated data demonstrated noteworthy differences in concentration in the polyphenol groups for the three different wines (grape cultivars). However, only subtle differences in certain individual polyphenol 


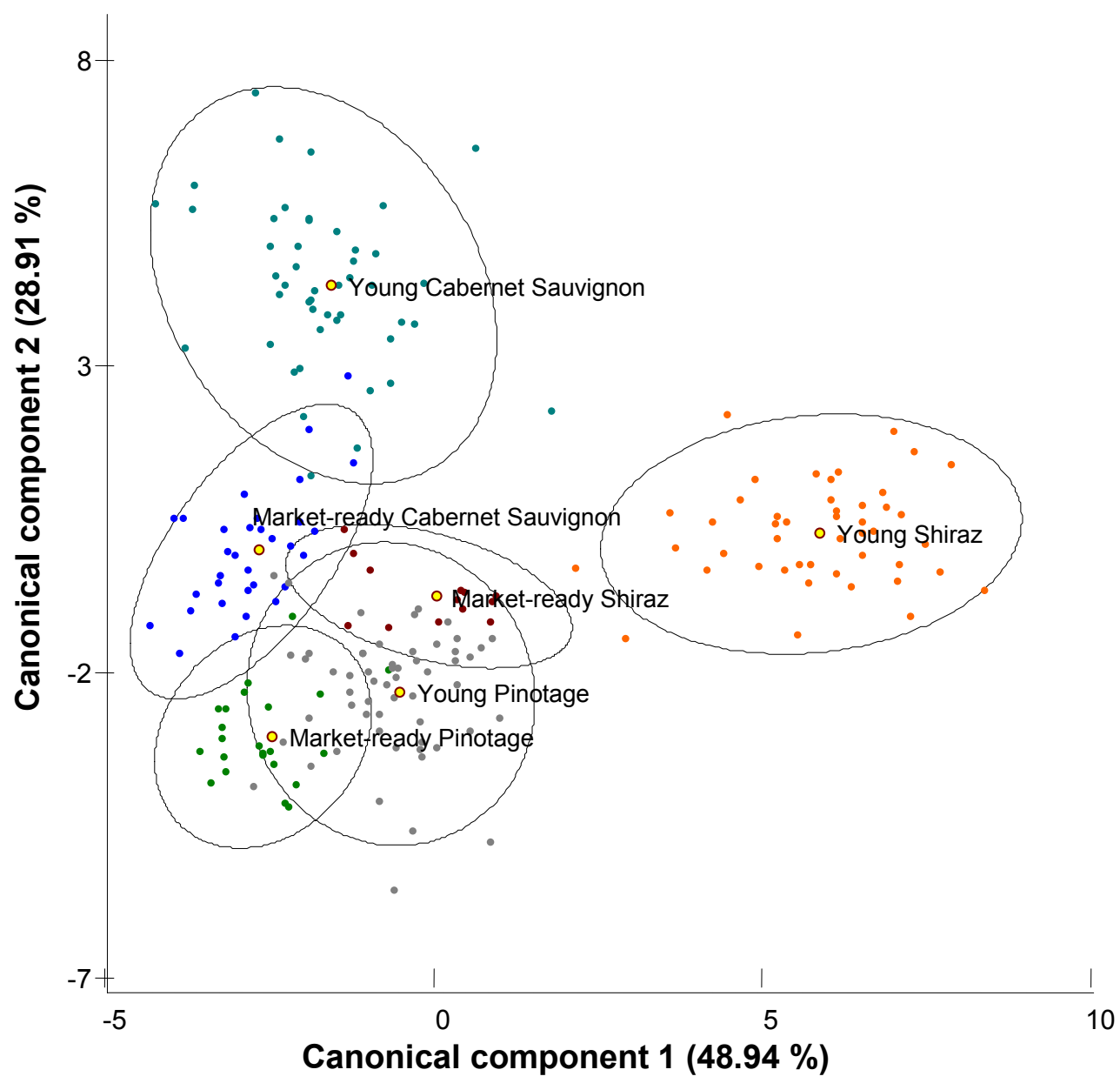

FIGURE 3

Plots of the first two canonical variables (Function 1 and Function 2) for market-ready and young Cabernet Sauvignon, Pinotage and Shiraz wines showing differentiation based on the concentrations of peonidin glucoside acetate, petunidin glucoside $p$-coumarate, malvidin glucoside acetate, delphinidin glucoside $p$-coumarate, malvidin glucoside $p$-coumarate, peonidin 3-O-glucoside, catechin, quercetin-3 glucoside, tryptophol, isorhamnetin and quercetin aglycone.

TABLE 4

Total sample standardised canonical coefficients for market-ready and young Pinotage, Cabernet Sauvignon and Shiraz wines.

\begin{tabular}{lcc}
\hline \multicolumn{1}{c}{ Variable } & Function 1 & Function 2 \\
\hline Gallic acid & -0.282 & -0.022 \\
Delphinidin glucoside acetate & 0.071 & 0.318 \\
Peonidin glucoside acetate & 0.701 & 0.218 \\
Petunidin glucoside $p$-coumarate & 0.895 & 0.153 \\
Malvidin 3- $O$-glucoside & 0.362 & 0.243 \\
Petunidin glucoside acetate & 0.239 & 0.414 \\
Malvidin glucoside acetate & 0.293 & 0.529 \\
Petunidin 3- $O$-glucoside & 0.331 & 0.128 \\
Delphinidin glucoside $p$-coumarate & 0.840 & 0.042 \\
Malvidin glucoside $p$-coumarate & 0.828 & 0.237 \\
Cyanidin 3- $O$-glucoside & 0.003 & 0.376 \\
$p$-Coumaric acid & -0.095 & -0.281
\end{tabular}


TABLE 4 (CONTINUED)

Total sample standardised canonical coefficients for market-ready and young Pinotage, Cabernet Sauvignon and Shiraz wines.

\begin{tabular}{lcc}
\hline \multicolumn{1}{c}{ Variable } & Function 1 & Function 2 \\
\hline Delphinidin 3- $O$-glucoside & 0.061 & 0.420 \\
Peonidin 3-O-glucoside & 0.538 & 0.247 \\
Epicatechin & 0.417 & 0.305 \\
Catechin & 0.070 & 0.594 \\
Pinotin A & -0.149 & -0.367 \\
Polymeric pigments & 0.184 & 0.188 \\
Quercetin-3 glucoside & 0.680 & 0.056 \\
Tryptophol & 0.146 & 0.583 \\
Protocatechuic acid & -0.026 & 0.015 \\
Quercetin aglycone & 0.576 & 0.448 \\
Epicatechin gallate & -0.137 & 0.226 \\
Isorhamnetin & 0.708 & 0.303 \\
Vitisin A & -0.096 & 0.247 \\
Coutaric acid & 0.183 & -0.089 \\
Polymeric phenols & 0.252 & 0.013 \\
Kaempferol & 0.265 & 0.420 \\
Caffeic acid & -0.140 & -0.479 \\
Epigallocatechin gallate & 0.126 & -0.336 \\
Procyanidin B1 & 0.179 & -0.214 \\
Caftaric acid & -0.022 & -0.386 \\
\hline
\end{tabular}

compound concentrations among the three grape cultivars were detected. These subtle differences are indicative of wines that were randomly analysed according to grape cultivar. Grape origin, terroir and winemaking techniques, which influence the polyphenol content of grapes, were not considered as parameters.

\section{LITERATURE CITED}

Arvanitoyannis, I.S., Katsota, M.N., Psarra, E.P., Soufleros, E.H. \& Kallithraka, S., 1999. Application of quality control methods for assessing wine authenticity: Use of multivariate analysis (chemometrics). Trends Food Sci. Tech. 10, 321-336.

Burns, J., Mullen, W., Landrault, Teissedre, P-L., Lean, E. J. \& Crozier A., 2002. Variations in the Profile and Content of Anthocyanins in Wines Made from Cabernet Sauvignon and Hybrid Grapes. J. Agric. Food Chem. 50, 4096-4102.

Cliff, M. A., King, M.C. \& Schlosser, J., 2007., Anthocyanin, Phenolic composition, colour measurement and sensory analysis of $\mathrm{BC}$ commercial red wines. Food Res. Int. 40, 92-100.

De Villiers, A., Majek, P., Lynen, F., Crouch, A., Lauer, H. \& Sandra, P., 2005. Classification of South African red and white wines according to grape variety based on the non-coloured phenolic content. Eur. Food Res. Technol. 221, 520-528.

Fang, F., Li, J-M., Zhang, P., Tang, K., Wang, W., Pan, Q-H. \& Huang, W-D., 2008. Effects of grape variety, harvest date, fermentation vessel and wine ageing on flavonoid concentration in red wines. Food Res. Int. 41, 53-60.

Ferrer-Gallego, R., García-Marino, M., Hernández-Hierro, J-M., RivasGonzalo, J-C \& Escribano-Bailón, M. T., 2010. Statistical correlation between flavanolic composition, colour and sensorial parameters in grape seed during ripening. Anal. Chim. Acta. 660, 22-28.
García-Beneytez, E., Cabello, F. \& Revilla, E., 2003. Analysis of grape and wine anthocyanins by HPLC-MS. J. Agric. Food Chem. 51, 5622-5629.

Goldberg, D.M., Karumanchiri, A., Tsang, E. \& Soleas, G.J., 1998a. Catechin and epicatechin concentrations of red wines: regional and cultivarrelated differences. Am. J. Enol. Vitic. 49, 23-34.

Goldberg, D.M., Tsang, E., Karumanchiri, A. \& Soleas, G.J., 1998b. Quercetin and $p$-coumaric acid concentrations in commercial wines. Am. J. Enol. Vitic. 49, 142-151.

Jaitz, L., Siegl, K., Eder, R., Rak., G., Abranko, L., Koellensperger, G. \& Hann, S., 2010. LC-MS/MS analysis of phenols for classification of red wine according to geographic origin, grape variety and vintage. Food Chem. $22,366-372$.

Kallithraka, S., Tsoutsouras, E., Tzourou, E. \& Lanaridis, P., 2006. Principal phenolic compounds in Greek red wines. Food Chem. 99 (4), 784-793.

Makris, P.D., Kallithraka, S. \& Mamalos, A., 2006. Differentiation of young red wines based on cultivar and geographical origin with application of chemometrics of principal polyphenolic constituents. Talanta 70, 1143 1152

OIV (Office International de la Vigne et du Vin), Resolution Oeno 22/2003, Paris, 2003.

Otteneder, H., Marx, R. \& Zimmer, M., 2004. Analysis of the anthocyanin composition of Cabernet Sauvignon and Portugieser wines provides an objective assessment of the grape variety. Aust. J. Grape \& Wine Res. 10, 3-7.

Rastija, V., Srečnik, G. \& Medić-Šarić, M., 2009. Polyphenolic composition of Croation wines with different geographical origins. Food Chem. 115, 5460

Rencher, A.C., 2002 ( $2^{\text {nd }}$ Ed). Methods of Multivariate Analysis. John Wiley \& Sons. 
Revilla, E., Garcia-Beneytez, E., Cabello, F., Martin-Ortega, G. \& Ryan, J-M., 2001. Value of high-performance liquid chromatographic analysis of anthocyanins in the differentiation of red grape cultivars and red wines made from them. J. Chrom. A. 915, 53-60.

Ribéreau-Gayon, M.W., Glories, Y., Maujean, A. \& Du Bourdieu, D., 2006. Phenolic Compounds. In: Handbook of Enology. The Chemistry of Wine Stabilisation and Treatments, vol. 1. John Wiley \& Sons, New York. pp. $129-185$.

Rossouw, M. \& Marais, J., 2004. The phenolic composition of South African Pinotage, Shiraz and Cabernet Sauvignon wines. S. Afr. J. Enol. Vitic. 25, 94-04.

Ryan, J-M. \& Revilla, E., 2003. Anthocyanin Composition of Cabernet Sauvignon and Tempranillo Grapes at Different Stages of Ripening. Agric. Food Chem. 51, 3372-3378.

Schwarz, M., Quast, P., Von Baer, D. \& Winterhalter, P., 2003. Vitisin A Content in Chilean Wines from Vitis vinifera $\mathrm{Cv}$. Cabernet Sauvignon and Contribution to the Colour of Aged Red Wines. J. Agric. Food Chem. 51, 6261-6267.
Von Baer, D., Mardones, C., Gutierrez, L., Hofmann, G., Becerra, J., Hitschfeld, A. \& Vergara, C., 2005. Varietal authenticity verification of Cabernet Sauvignon, Merlot and Carmenère wines produced in Chile by their anthocyanin, flavonol and shikimic acid profiles. Bulletin De L'O.I.V. (887-888), 45-57.

Von Baer, D., Rentzsch, M., Hitschfeld, M. A., Mardones, C., Vergara, C. \& Winterhalter, P., 2008. Relevance of chromatographic efficiency in varietal authenticity verification of red wines based on their anthocyanin profiles: Interference of pyranoanthocyanins formed during wine ageing. Anal. Chim. Acta. 621, 52-56.

Waterhouse, A.L., Price, S.F. \& McCord, J.D., 1999. Reversed-phase high-performance liquid chromatography methods of analysis of wine polyphenols. Meth. Enzym. 299, 113-21.

Zoecklein, B.W., Fugelsang, K.C., Gump, B.H. \& Nury, F.S., 1999. Phenolic compounds and wine colour. In: Production Wine Analysis, New York. pp. $129-168$. 